\title{
Case-crossover design in air pollution epidemiology
}

\author{
J.J.K. Jaakkola
}

\begin{abstract}
Case-crossover design in air pollution epidemiology. J.J.K. Jaakkola. C)ERS Journals
\end{abstract} Ltd 2003.

ABSTRACT: The case-crossover design was developed to study the effects of transient, short-term exposures on the risk of acute events, such as myocardial infarction, in the early 1990s. This paper illustrates how the principles of case-crossover design are related to the principles of crossover and case-control designs and stipulates the possibilities of case-crossover design in air pollution epidemiology.

In the case-crossover design, the study population consists of subjects who have experienced an episode of the health outcome of interest. Similar to a crossover study, each subject serves as his or her own control. As in a matched case-control study, the inference is based on a comparison of exposure distribution rather than the risk of disease. The case-crossover study is most suitable for studying relations with the following characteristics: 1) the individual exposure varies within short time intervals; 2) the disease has abrupt onset and short latency for detection; and 3) the induction period is short.

Case-crossover design allows use of routinely monitored air pollution information and at the same time makes it possible to study individuals rather than days as the unit of observation. Case-crossover design is amenable for studying the effects of varying short-term air pollution exposure on health outcomes with an abrupt onset, such as myocardial infarction or asthma attack.

Eur Respir J 2003; 21: Suppl. 40, 81s-85s.

\author{
Correspondence: J.J.K. Jaakkola \\ Institute of Occupational Health \\ University of Birmingham \\ Edgbaston \\ Birmingham \\ B15 2TT \\ UK \\ E-mail: j.jaakkola@bham.ac.uk \\ Fax: 358919127570
}

\author{
Keywords: Air pollution \\ epidemiologic methods \\ case-crossover study \\ case-control study \\ crossover study
}

Received and accepted: April 122002
Air pollution may influence human health through various biological mechanisms causing diverse health effects [1, 2]. Different types of exposure patterns of a given air pollutant, such as duration and intensity of exposure over time, may induce an onset of a disease process which, after a shorter or longer duration of time, leads to a manifestation of a disease. For example, short-term exposure to high levels of carbon monoxide (CO) may trigger an acute myocardial infarction (MI) within minutes from the beginning of the exposure, whereas causation of lung cancer may require a long-term exposure. Epidemiological studies provide important empirical evidence of increased health risks related to air pollution exposure [3]. The study design influences strongly the credibility of causal inference from the results. The choice of the study design should depend primarily on the type of hypothesised relation between exposure and outcome to be studied. Different approaches are needed to study effects of short-term and long-term exposure, and the type of health effect.

The first observations of the effects of short-term exposure to air pollution were made during very high levels of air pollution in Meuse Valley, Belgium in 1930 [4] and in London in the 1950s [5]. The first time-series analyses of registry-based morbidity and mortality information and air pollution monitoring data were conducted in the 1970s. In the 1990s, Poisson regression analysis and sophisticated modelling techniques were developed to take into account long-term seasonal and secular trends $[3,6]$.

The case-crossover design was developed by MACLURE [7] to study effects of transient short-term exposures on the risk of acute events, such as MI in the early 1990s. Recently casecrossover design has been applied as a complementary or alternative approach for studying effects of short-term exposure to air pollution $[8,9]$. The objective of this presentation is to illustrate the principles of case-crossover design by comparing it with crossover and case-control designs. The possibilities of case-crossover in studying effects of short-term exposure to air pollution on acute health outcomes such as MI or asthma attack will also be discussed.

\section{The principles of case-crossover design}

In the case-crossover design, the study population consists of subjects who have experienced an episode of the health outcome of interest. All the study subjects are cases, individuals who have experienced the outcome of interest. The design focuses on the point in time when the event occurred. Therefore this design is applicable to the outcomes whose onset can be defined. The case-crossover design bears resemblance to both a classic crossover study and a matched case-control study. Similar to a crossover study, each subject serves as his or her own control. As in a matched case-control study, the inference is based on a comparison of exposure distribution rather than the risk of disease. All these three study designs have a similar analytic approach. Figures 1-3 illustrate the basic designs and inferences of crossover, matched case-control and case-crossover studies.

\section{Crossover study}

Crossover trial is an experimental study design where the investigator changes the exposure according to the interests of the study hypothesis. Consider a simplified situation where the effect of air pollution exposure is assessed based on the occurrence of asthma attacks among asthmatic subjects. Exposure is considered as being present or absent. In a basic crossover trial, group 1 is first exposed during a hazard period 
Design

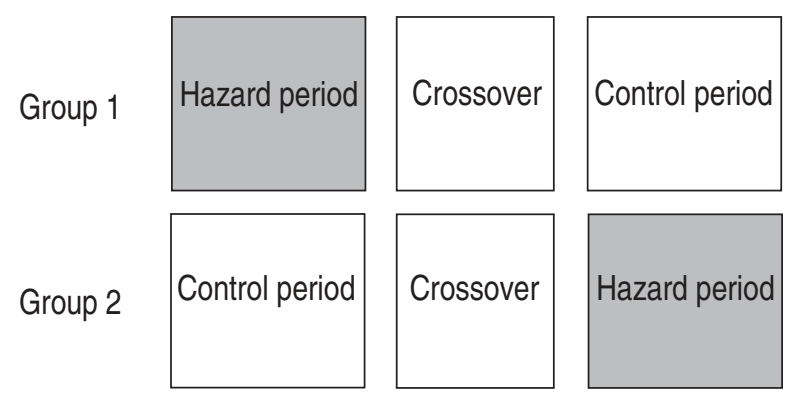

Inference

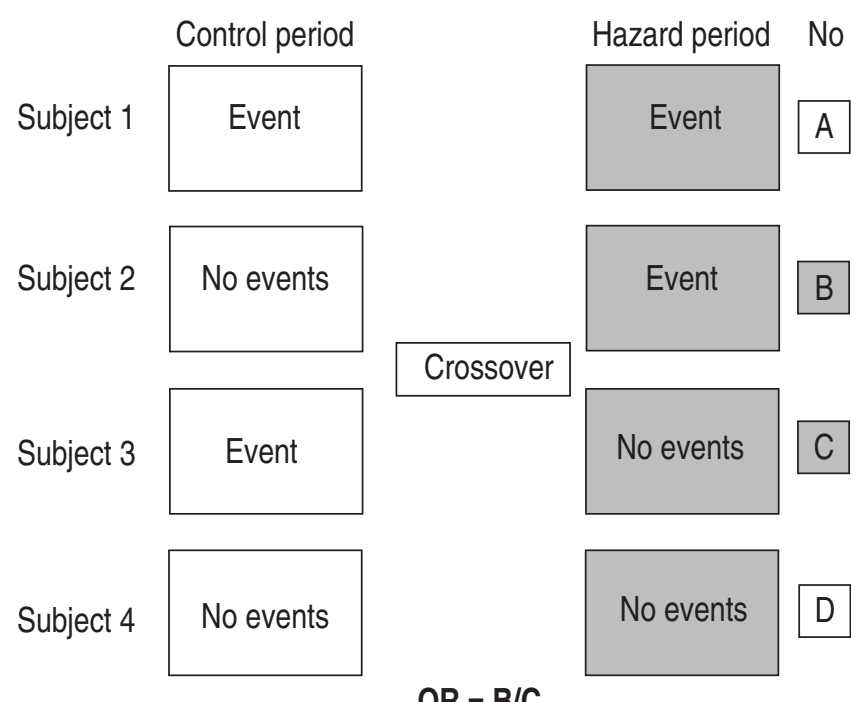

Fig. 1.- The design of the crossover trial and schematic presentation of the scientific inference. No: number; OR: odds ratio.

(called a treatment period in clinical crossover trials) while group 2 remains unexposed during a control period (fig. 1). During a crossover exposure conditions are switched and now group 2 undergoes the hazard period and group 1 is unexposed. The inference on the effect of exposure is based on a comparison of each subject's outcome experience during the hazard and control periods. In the example of the study of asthmatics, the participants experience a hazard period of exposure to air pollution and a control period of low or no exposure and the outcome of interest is an episode of asthma attack (fig. 1, inference). Subject 1 experiences an asthma attack during both the control and exposure period and Subject 4 does not have any attacks, either during the hazard or during the control period. Subject 2 has an attack during the hazard period but not during the control period and Subject 3 during the control period but not during the hazard period. Subjects 1 and 4 do not contribute any knowledge of the effect of exposure, while the relative number of subjects of type 2 (B) and type 3 (C) are used to assess the effect calculating odds ratio by dividing B by $\mathrm{C}$. Crossover study is suitable for exposure-effect relations with a short induction period and reversible effects.

The current author's group has used crossover trials in air pollution epidemiology to assess the roles of air recirculation [10] and air humidification [11] in the office-building environment in the causation of symptoms and signs of sick building syndrome. The first study tested the hypothesis that re-circulated air in mechanically ventilated office buildings causes symptoms commonly referred to as the sick building syndrome

\section{Design}

Study base

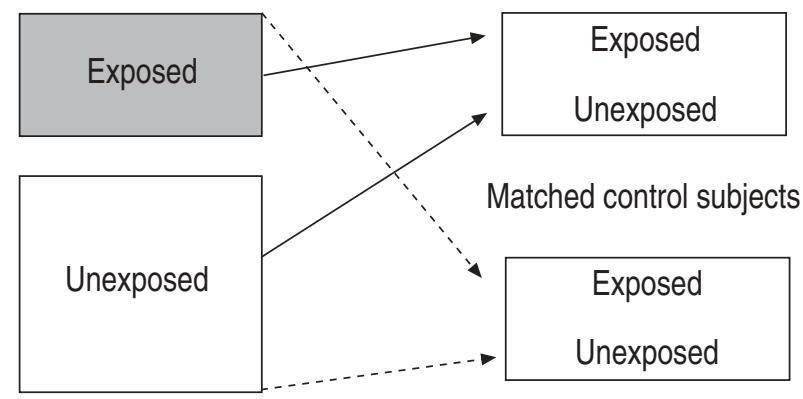

Inference
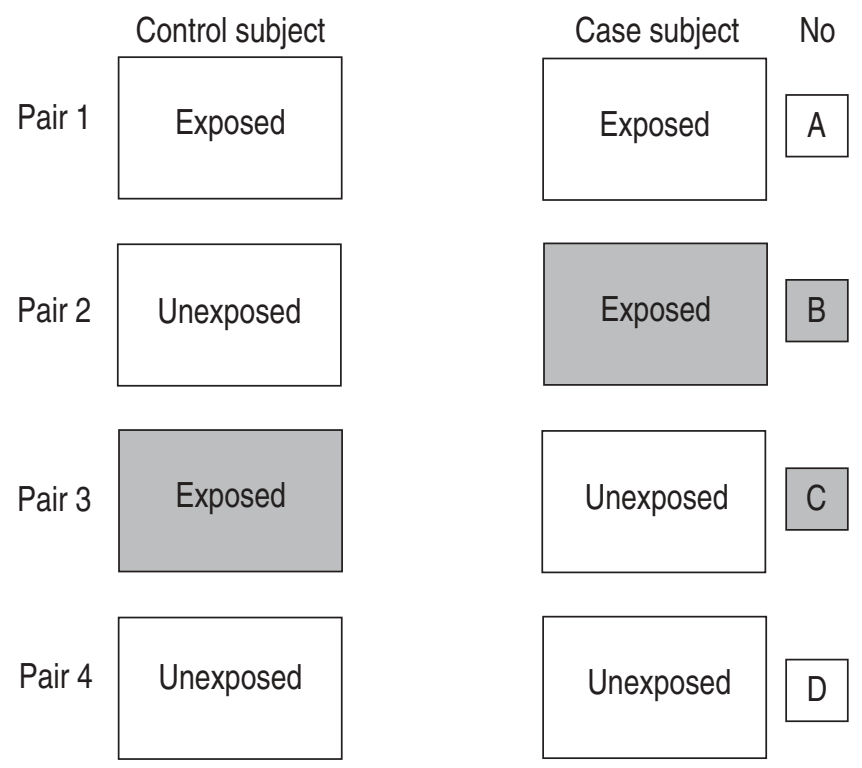

\section{$\mathrm{OR}=\mathrm{B} / \mathrm{C}$}

Fig. 2. - The design of the matched case-control study and a schematic presentation of the scientific inference. No: number; OR: odds ratio.

[10]. A blinded four-period crossover trial was conducted in two similar office buildings (Building 1 and Building 2) with a total airflow of $20 \mathrm{~L} \cdot \mathrm{s}^{-1}$ per person, contrasting $70 \%$ re-circulated air (hazard period) with $0 \%$ of re-circulated air (control period). Each period lasted 1 work-week. During the first week, workers in Building 1 were exposed to $70 \%$ recirculated air, whereas no air recirculation was used in Building 2. Each weekend the air recirculation was switched in the buildings from 70 to $0 \%$ or vice versa. Participants reported their ratings of symptoms, their perceptions of indoor air quality and other relevant information in a daily diary. For each individual, the outcome experience during the hazard and control period was compared. In the statistical analyses, the individuals were grouped analogously to figure 1 . Subjects who reported an equal amount of symptoms during the hazard and control periods (A) or no symptoms in either period (D) did not contribute any information. The basic inference was based on the number of subjects who experienced more symptoms during the hazard periods (B) divided by the number of subjects who experienced more symptoms during the control period (C). For example, there were 22 subjects (B) with more nasal dryness during the hazard periods than during the control periods and 15 subjects with more nasal dryness during the control period, whereas the rest of the participants had an equal amount (A) or no symptoms (D) in 


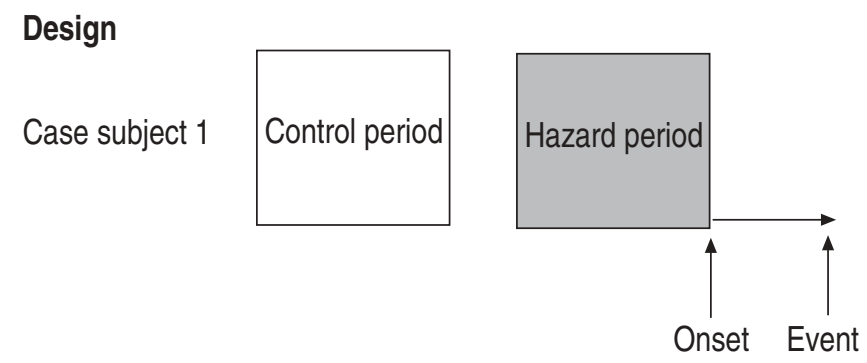

Inference

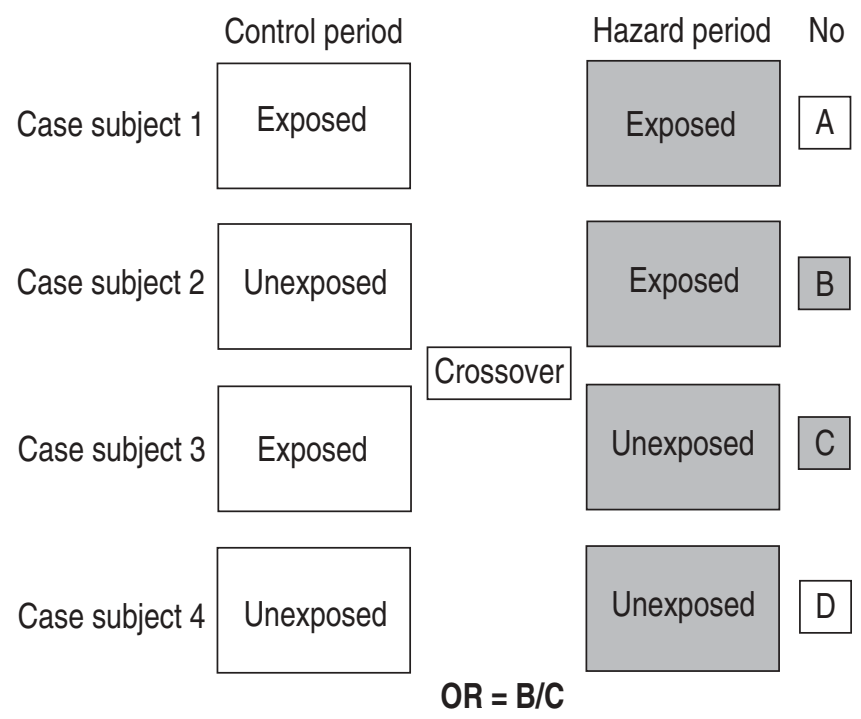

Fig. 3. - The design of case-crossover study and schematic presentation of the scientific inference. No: number; OR: odds ratio.

the hazard and control periods. The risk estimate was calculated as $22 / 15=1.47$ with a $95 \%$ confidence interval (CI) $0.76-2.83$. Because the inference was based on intra-individual comparison of symptoms during the hazard and control periods, i.e. each person served as his or her own control, there was no need for adjustment for confounding. The current author's group carried out a second six-period crossover trial to assess whether air humidification in the office environment alleviates the symptoms of the sick building syndrome [11].

\section{Matched case-control study}

In a case-control study, cases of the disease of interest are selected from a study base and controls are selected from the same study base that produced the cases. In a matched pair design, for each case a control is chosen who is similar according to selected matching factors, such as age, sex or other potential determinants of the studied disease (fig. 2). The inference is based on a comparison of the exposure between the case and control subjects of each pair. Again, pairs with similar exposure status (present or absent) do not contribute information for the inference. The measure of effect is based on the pairs discordant for exposure, and the effect is quantified by dividing the number of pairs with an exposed case (B) by the number of pairs with an exposed control (C). The casecontrol design is suitable for assessing the relation with both short and long induction period.

The current author's group used a matched case-control study to assess the effects of various environmental exposures on the risk of bronchial obstruction in Norwegian children
[12-16]. A cohort of 3,754 newborns in Oslo was recruited for the study. The children were followed and all the children with symptoms and signs of bronchial obstruction during the first 2 yrs of life were identified as cases. A control matched for age was chosen for each case. Exposure assessment was conducted within 1 week from the first contact concurrently in the homes of the case and the control. The study population consisted of 251 case-control pairs. In the study of the relation between dampness problems and the risk of bronchial obstruction, a trained investigator evaluated the homes with dampness problems. In 12 case-control pairs (A) both the case and the control were found to be exposed to dampness and in 160 case-control pairs (D) both the case and the control were unexposed. In 57 pairs (B) the case but not the control was exposed and in 22 (C) pairs only the control was exposed. Thus the crude odds ratio was 57/22=2.6 (1.6-4.2 95\% CI). Conditional logistic regression analysis was applied to adjust for potential confounders such as the child's sex, presence of siblings, day-care attendance, duration of breastfeeding, exposure to environmental tobacco smoke, and parental atopy. The adjustment had little influence on the risk estimate.

\section{Case-crossover study}

In a case-crossover design, the study subjects are selected from cases, i.e. those who have experienced an event of interest, for example an episode of MI. Similar to a crossover trial, each study subjects serves as his/her own control. In contrast to the crossover trial, in the case-crossover study the investigator does not influence or control the exposure of interest. In the case-control study, the controls are selected to represent the usual exposure levels in the source population that produced the cases.

A hazard period is the time period for which the exposure status or level is defined. It should represent the average time period of exposure in the population, which is relevant for the causation of the disease. The hazard period tends to be imprecise, because it incorporates variation in individual induction times, as well as uncertainty of the timing of the exposure and onset of disease. For the relation between ambient air $\mathrm{CO}$ and acute MI, tentative hazard periods could be applied from 6-24 hrs prior to the onset of angina pain.

In air pollution studies, where exposure assessment is based on stationary air pollution monitoring, bi-directional control periods, i.e. before and after the event, offer an attractive option (fig. 4). Bi-directional sampling of control periods helps to adjust for seasonal trends in exposure levels.

The inference is based on a comparison of each subject's exposure during a time period relevant for the causation of the outcome, often referred to as a hazard period, and during one or more control periods. The analytic approach is analogous to crossover and matched case-control studies. In the basic inference, for each case the exposure status during the hazard and control periods are compared, and only subjects with different levels of exposure are informative. The measure of effect, called the odds ratio is calculated by dividing the number of subjects exposed only during the hazard period by those exposed during the control period, as illustrated in figure 3 . The methods for calculating CIs using both exact method and a large sample approach are described by RoTHMAN [17]. Conditional logistic regression analysis can be used to estimate adjusted odds ratios. Conditional logistic regression analysis allows modelling using several or a varying number of control periods. Exposure can be characterised quantitatively using the level, cumulative exposure, exposure time, exposure profile or a meaningful combinations of these. 


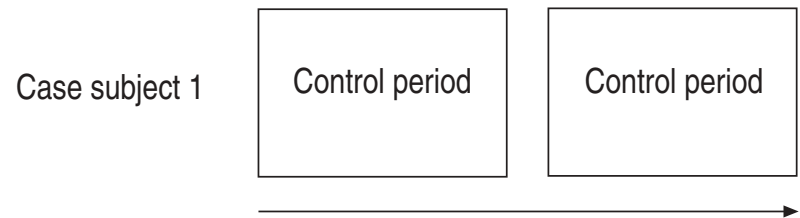

Time
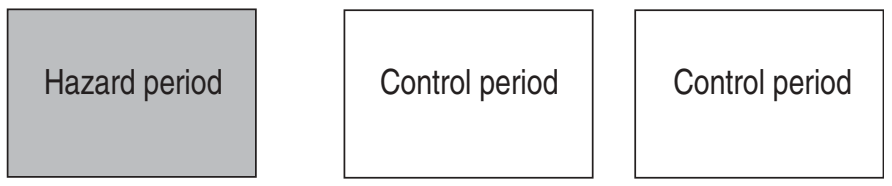

Fig. 4.-Bidirectional sampling of control time in the case-crossover study.

\section{Study questions and causal hypotheses}

The case-crossover study is most suitable for studying relations with the following characteristics 1) the individual exposure varies within a short time-interval; 2) the disease has abrupt onset and short latency for detection; and 3) the induction period is short $[18,19]$.

To illustrate these issues, consider the relation between exposure to $\mathrm{CO}$ and fatal MI. 1) CO is produced by the incomplete combustion of carbon-containing fuels:

$$
2 \mathrm{C}+\mathrm{O}_{2} \rightarrow 2 \mathrm{CO}
$$

Automobile engines are the major outdoor source of $\mathrm{CO}$ in urban areas, and the levels of $\mathrm{CO}$ vary substantial according to traffic density within a given day and from day-to-day [20]. 2) MI leading to death evolves usually within hours from the onset of myocardial ischaemia experienced as angina pain to irreversible damage of myocardial muscle, cardio-respiratory failure and death. The onset of symptoms can be considered operationally as the onset of the disease event. 3) CO is known to have a high capacity to block the oxygen receptor of haem and induce myocardial ischaemia, and therefore the induction period from the beginning of the exposure to the onset of disease of likely to be short. The length of induction period rather depends on the exposure concentrations, and in the context of air pollution, the question is whether levels encountered in the environment have a capacity to increase the risk of fatal MI. It is likely that long-term exposure to low levels of $\mathrm{CO}$ increase the risk of MI by causing chronic hypoxia, which may contribute to the development of coronary heart disease, but this type of relation does not suit assessment in a casecrossover study. In conclusion, the relation between short-term exposure to $\mathrm{CO}$ and risk of fatal MI appears amenable to be assessed in a case-crossover study.

A causal hypothesis suitable to be tested in a case-crossover study could be generally formulated: does short-term exposure to CO cause MI? Or in stochastic terms: does this exposure increase the risk of MI? MACluRE and MitTElman [19] suggest that the best way to express the causal hypothesis is to focus on exposed cases and use a counterfactual condition statement, which for this relation would be: some exposed MIs would not have occurred at that time, if they had not been exposed to unusually high ambient air $\mathrm{CO}$ immediately before the event. The control period(s) represent the usual levels of exposure whereas the hazard period preceding the onset of the disease process represents the exposure of interest. According to a causal hypothesis, among the individuals having experienced the MI the exposure levels during the hazard periods should on average be higher than the exposure levels during the control periods.

\section{Strengths and limitations of case-crossover design in air pollution epidemiology}

In case-crossover study, cases serve as their own controls and therefore the design eliminates confounding by stable individual characteristics. Case-crossover design allows use of routinely monitored air pollution information and at the same time makes it possible to study individuals rather than days as the unit of observation.

There are two advantages compared with studies based on daily counts and air pollution levels. First, information on individual characteristics such as age, sex, health status and behavioural factors make it possible to study effect modification i.e. to identify individuals susceptible to the effects of air pollution. Secondly, bi-directional selection of control periods allows individual adjustment for seasonal and secular trends. A disadvantage of this approach is that compared with Poisson regression time-series analysis is approximately 50\% lower power, as shown by BATESON and SCHWARTZ [21].

Case-crossover design in air pollution epidemiology is amenable for studying the effects of varying short-term air pollution exposure on health outcomes with an abrupt onset, whereas other types of study are needed for studying effects of long-term exposure.

\section{References}

1. American Thoracic Society. Committee on environmental and occupational health assembly. Health effects of outdoor air pollution. Part 1. Am J Respir Crit Care Med 1996; 153: 3-50.

2. American Thoracic Society. Committee on environmental and occupational health assembly. Health effects of outdoor air pollution. Part 2. Am J Respir Crit Care Med 1996; 153: 477-498.

3. Samet JM, Jaakkola JJK. Epidemiologic methods. In: Holgate ST, Samet JM, Koren HS, Maynard RL, eds. Air Pollution and Health. London, Academic Press, 1999; pp. 429-460.

4. Nemery B, Hoet PHM, Nemmar A. The Meuse Valley of 1930: an air pollution disaster. Lancet 2001; 357: 704-708.

5. Logan WPD. Mortality in London for incident. Lancet 1953; 1: 336-338.

6. Pope CA, Dockery DW. Epidemiology of particle effects. In: Holgate ST, Samet JM, Koren HS, Maynard RL, eds. Air Pollution and Health. London, Academic Press, 1999; pp. 473-705.

7. Maclure M. The case-crossover design: a method for studying transient effects on the risk of acute events. $\mathrm{Am} \mathrm{J}$ Epidemiol 1991; 133: 144-153.

8. Lee JT, Schwartz J. Reanalysis of the effects of air pollution on daily mortality in Seoul, Korea: A case-crossover design. Environ Health Perspect 1999; 107: 633-636.

9. Neas LM, Schwartz J, Dockery D. A case-crossover analysis of air pollution and mortality in Philadelphia. Environ Health Perspect 1999; 107: 629-631.

10. Jaakkola JJK, Tuomaala P, Seppänen O. Air recirculation and sick building syndrome: a blinded crossover trial. Am J Public Health 1994; 84: 422-428.

11. Reinikainen LM, Jaakkola JJK, Seppänen O. The effect of air humidification on symptoms and the perception of air quality in office workers. A six period cross-over trial. Arch Environ Health 1992; 47: 8-15. 
12. Nafstad P, Øie L, Mehl R, et al. Residential dampness problems and development of bronchial obstruction in Norwegian children. Am J Respir Crit Care Med 1998; 157: 410-414.

13. Jaakkola JJK, Øie L, Nafstad P, Botten G, Samuelsen SO, Magnus P. Surface materials in the home and development of bronchial obstruction in young children in Oslo, Norway. Am J Publ Health 1999; 84: 188-192.

14. Øie L, Nafstad P, Botten G, Magnus P, Jaakkola JJK. Ventilation in the homes and bronchial obstruction in young children. Epidemiology 1999; 10: 294-299.

15. Nafstad P, Magnus P, Gaarder PI, Jaakkola JJK. Early life exposure to pets and atopy related diseases in the first 4 years of life. Allergy 2001; 56: 307-312.

16. Jaakkola JJK, Nafstad P, Magnus P. Environmental tobacco smoke, parental atopy, and childhood asthma. Environ Health Perspect 2001; 109: 579-582.

17. Rothman KJ. Modern epidemiology. Boston/Toronto, Little, Brown and Company, 1986.

18. Rothman KJ, Greenland S. Case-control studies. In: Rothman KJ, Greenland S, eds. Modern Epidemiology. 2nd Edn. Philadelphia, Lippincott-Raven, 1998.

19. Maclure M, Mittleman MA. Should we use a case-crossover design? Annu Rev Public Health 2000; 21: 193-221.

20. Maynard RL, Waller R. Carbon monoxide. In: Holgate ST, Samet JM, Koren HS, Maynard RL, eds. Air pollution and health. London, Academic Press, 1999; pp. 749-796.

21. Bateson TF, Schwartz J. Control for seasonal variation and time trend in case-crossover studies of acute effects of environmental exposures. Epidemiology 1999; 10: 539-544. 